\title{
Aliança terapêutica, vinculação parental e sintomatologia de pacientes adultos que iniciam psicoterapia
}

\section{Associations between therapeutic alliance, parental bonding and symptomatology in adult patients beginning psychotherapy}

\section{Allianza Terapéutica, vinculación parental y sintomatología del pacientes adultos que comienzan la psicoterapia}

\section{Carolina Palmeiro Lima*}

Universidade do Vale do Rio dos Sinos - UNISINOS, São Leopoldo, Rio Grande do Sul, Brasil

\section{Fernanda Barcellos Serralta**}

Universidade do Vale do Rio dos Sinos - UNISINOS, São Leopoldo, Rio Grande do Sul, Brasil

\begin{abstract}
RESUMO
A aliança terapêutica (AT) é uma variável chave para o processo de mudança, sendo afetada por fatores do terapeuta e do paciente. Este estudo investigou a relação entre AT, vinculação parental e sintomatologia de pacientes em Psicoterapia Psicanalítica. Foram examinados 57 pacientes adultos entre a $4^{a}$ e a $5^{a}$ sessão de tratamento com medidas de avaliação da AT, da vinculação com os pais na infância e adolescência e de sintomas psicopatológicos. Associações positivas entre AT e cuidados da mãe e associações negativas entre AT e controle da mãe foram encontradas. Além disso, foram constatadas diferenças na AT entre os estilos parentais "cuidado ótimo" e "controle sem afeto" da mãe. Não houve associação entre AT e sintomas. Por outro lado, diversos sintomas mostraram associação positiva com o controle do pai. Os resultados indicam que a AT está relacionada com a história pregressa de vinculação do paciente e esta história tem relação com seu quadro clínico atual, evidenciando a complexidade dos processos que ocorrem na psicoterapia.

Palavras-chave: psicoterapia, AT, relação, vinculação, sintomatologia.
\end{abstract}

\section{ABSTRACT}

The Therapeutic Alliance (TA) is a key-variable for the process change, and it's affected by therapist's and patients' factors. This study investigated the relationship between TA, parental bonding and symptomatology in patients in psychoanalytic psychotherapy. Fifty-seven adult participants were examined between the $4^{\text {th }}$ and the $5^{\text {th }}$ session of the treatment with assessments of TA, child and adolescence parental bonding and psychopathological symptoms. Positive associations between TA and 
"mother care" and negative associations between "mother overprotection" and TA were found. Also, significant differences were found in TA and the mother's parenting styles "optimal parenting" and "affectionless control". TA and symptoms were not associated. However, several symptoms had positive associations with father overprotection. Results indicate that TA is related with the parental bonding history and that this history is related with patients' current clinical condition, showing the complexity of the processes that occur in psychotherapy.

Keywords: psychotherapy, TA, relationship, bonding, symptomatology.

\section{RESUMEN}

La Alianza Terapéutica (AT) es una variable clave para el proceso de cambio y es afectada por factores del terapeuta y del paciente. Este estudio examinó la relación entre la AT, la vinculación parental y la sintomatología en pacientes en psicoterapia psicoanalítica. Se examinaron cincuenta y siete participantes adultos entre la cuarta y la quinta sesión del tratamiento con instrumentos de evaluación de la alianza terapéutica, de la vinculación parental en la infancia y adolescencia, y de los síntomas psicopatológicos. Se encontraron asociaciones positivas entre la AT y el cuidado de la madre, y asociaciones negativas entre la AT e la protección de la madre. También se constató diferencias significativas en la AT y los estilos parentales de la madre "cuidado óptimo" y "control sin afecto". No hubo asociación entre AT y síntomas. Sin embargo, varios síntomas mostraran asociación positiva con el control del padre. Los resultados indican que la AT se relaciona con la historia anterior de vinculación del paciente y que esta historia tiene que ver con su cuadro clínico actual, mostrando la complejidad de los procesos que ocurren en la psicoterapia.

Palabras-clave: psicoterapia, TA, relación, vinculación, sintomatología.

\section{I ntrodução}

A psicoterapia tem um papel fundamental na vida das pessoas com sofrimento psicológico, pois é efetiva para uma ampla variedade de condições clínicas (Hofmann, Asnaani, Vonk, Sawyer \& Fang, 2012; Leichsenring \& Rabung, 2011). Um dos seus elementos centrais é a relação terapêutica, considerada pelas diversas abordagens terapêuticas como a base sobre a qual se desenvolve o processo terapêutico. Nesta relação, a aliança terapêutica ou aliança de trabalho (AT) é um dos aspectos mais estudados empiricamente.

Em sua origem, a AT remete ao conceito inicialmente definido por Freud (1912/1976) como transferência positiva. Porém, a criação do termo é atribuída a Elisabeth Zetzel (1956) que definiu a aliança como uma relação de trabalho que favorece o processo terapêutico e é capaz de trazer benefícios para este. Greenson (1965/2008) caracterizou a AT como uma parte objetiva e racional da relação com o terapeuta, principalmente no que diz respeito aos sentimentos da dupla. Diferentemente da transferência, a AT seria derivada de uma parte mais madura do ego, não envolvida no conflito, que emergiria da relação real (Eizirik, Libermann \& Costa, 2008). 
Embora o construto tenha origem na Psicanálise, hoje a AT é considerada um importante elemento do processo terapêutico de diversas abordagens de psicoterapia (Eizirik et al., 2008). Foi Bordin (1979) quem propôs que a aliança poderia ser compreendida por todas as psicoterapias. Segundo este autor, a AT contempla um vínculo colaborativo entre terapeuta e paciente e um acordo sobre objetivos e tarefas da terapia, definição amplamente aceita atualmente.

Diversos estudos evidenciaram a importância da AT para a clínica e a pesquisa, por constituir-se como um preditor significativo de resultados exitosos de psicoterapias de diferentes orientações teóricas (Arnow et al., 2013; Horvath, Del Re, Flückiger \& Symonds, 2011). Decorre destes estudos, o interesse de pesquisadores em examinar os fatores dos participantes que podem impactar 0 desenvolvimento e a manutenção desta variável no tratamento, bem como repercutir nos resultados. Um destes fatores é o apego entre pais e filhos. Na infância, é esta interação que conferirá à criança segurança para exploração do ambiente, e, por conseguinte, expectativas e confiança em torno de outras figuras de apego. Nesse sentido, o apego é fundamental para o funcionamento psicossocial da criança (Abreu, 2005a; Bowlby, 1984/2002) e influencia consideravelmente 0 desenvolvimento $e$ as relações futuras (Ainsworth, Blehar, Waters \& Wall, 1978/2001; Mikulincer \& Shaver, 2012).

Estudos também indicam que estabelecer laços emocionais e afetivos com alguém significativo e ser capaz de reparar eventuais rupturas promovem o desenvolvimento saudável da personalidade (Gao, Raine, Chan, Venables \& Mednick, 2010; Lewis, 2000). Logo, a psicoterapia seria um meio de facilitar a exploração de tais representações dos pacientes, o que possibilitaria mudanças ao longo do processo (Bowlby, 1984/2002). Ademais, o modo como muitos terapeutas lidam com seus pacientes aproxima-se de um relacionamento de base segura, ao serem, por exemplo, responsivos, consistentes e empáticos (Holmes, 2014).

$\mathrm{Na}$ literatura, diversas pesquisas objetivaram investigar estes relacionamentos no contexto da psicoterapia. Estudos procuraram compreender a relação entre AT e estilos de apego de pacientes (Diener \& Monroe, 2011; Marmarosh et al., 2009) e terapeutas (Degnan, Seymour-Hyde, Harris \& Barry, 2014). No que diz respeito ao paciente, uma meta-análise de 17 estudos empíricos revelou que o estilo de apego seguro está associado positivamente com a aliança, enquanto estilos inseguros possuem uma relação negativa com esta variável (Diener \& Monroe, 2011). Além disso, sintomas psicopatológicos têm sido relacionados com o apego inseguro, como por exemplo, depressão, ansiedade e hostilidade (Fortuna \& Roisman, 2008). 
Os padrões de apego são resultado das relações de vinculação precoces. Em se tratando dos vínculos parentais, a literatura revela associações entre esta variável e a AT. Num estudo com pacientes com anorexia $(n=30)$ ou bulimia $(n=27)$, alto suporte emocional paterno estava associado a uma maior qualidade da AT no grupo com anorexia. No grupo de pacientes com bulimia, a AT estava relacionada a um alto nível de suporte emocional da mãe e a uma baixa superproteção paterna (Tereno, Soares, Martins, Celani \& Sampaio, 2008).

Ademais, dificuldades no vínculo com pais ou cuidadores e experiências de apego adversas na infância (como perdas significativas ou situações traumáticas) estão relacionadas com um maior risco de desenvolver diversas psicopatologias na vida adulta (Morgan \& Fisher, 2007; Lima, Mello \& Mari, 2010; Campos, Besser \& Blatt, 2010). Já no que concerne à possível associação entre AT e sintomatologia, a literatura revela associação entre, por exemplo, sintomas típicos dos transtornos alimentares e AT, porém, com correlações fracas (Spokes, 2012). Já em um estudo brasileiro com adultos em psicoterapia de orientação analítica, não foram encontradas relações entre estas variáveis. (Gomes, 2003).

Embora a literatura internacional sustente algumas associações, a relação entre a AT e os estilos parentais dos pais, bem como a relação da sintomatologia com a AT parece não estar solidamente estabelecida. Ademais, no cenário nacional, uma revisão realizada nas bases SCIELO, LILACS e PEPSIC com as palavras-chave deste trabalho, revelou a escassez de estudos sobre o tema no país, sendo encontrado apenas um artigo que contempla as três variáveis principais. Entretanto, este versa sobre a relação com mudança em psicoterapia e os resultados foram inconclusivos devido às limitações do estudo (Abreu, 2005b). Logo, o propósito primário deste estudo é analisar a relação entre AT, vinculação parental e sintomatologia. Secundariamente, o estudo visa contribuir para a compreensão destas variáveis em amostras brasileiras.

\section{Materiais e Métodos}

Este é um estudo quantitativo, transversal e correlacional.

\section{Participantes}

No contexto de um estudo mais amplo, desde maio de 2015 estão sendo avaliados consecutivamente todos os pacientes com mais de 18 anos que estão na etapa inicial de psicoterapia psicanalítica (PP) em um ambulatório vinculado a um curso de formação em psicoterapia desta abordagem. No presente trabalho, os participantes 
são 57 pacientes adultos atendidos em PP por psicoterapeutas em formação ou já formados que estão vinculados ao ambulatório mencionado.

Instrumentos

WAI (Inventário de Aliança Terapêutica - Working Alliance Inventory) - Desenvolvido por Horvath e Greenberg (1994) tem como finalidade medir os três aspectos da aliança terapêutica propostos por Bordin (1979): tarefa, objetivos e o vínculo. É um inventário de auto resposta de 36 itens, do tipo Likert. A versão em português do Brasil foi desenvolvida mediante autorização do autor do instrumento original e os estudos estão em fase inicial não havendo dados de validade. Na amostra deste trabalho a confiabilidade baseada na homogeneidade (alpha de Cronbach) entre os itens do instrumento e das subescalas foi de 0.93 (escala total); 0.84 (tarefas); 0.74 (vínculo) e 0.83 (objetivos), sendo considerada satisfatória para as análises.

PBI (Instrumento de vinculação parental - Parental Bonding Instrument) - Desenvolvido por Parker, Tupling e Brown (1979/2011), é um instrumento de auto relato, do tipo likert. É composto de 25 afirmações em que o participante indica o quão parecido aquele comportamento é com os de seu pai e de sua mãe até os seus 16 anos. Mede dois construtos: afeto e controle (também chamado de superproteção). Altos escores na subescala "cuidado" indicam percepções de carinho e proximidade, enquanto que, em "controle" altos escores indicam proteção excessiva, vigilância e infantilização. Com base nos pontos de corte do instrumento original, é possível identificar diferentes estilos parentais: Cuidado Ótimo, Controle Afetivo, Controle sem Afeto, e Negligente. A versão em português do Brasil do PBI (Hauck et al., 2006), tem sua equivalência atestada, possuindo boa fidedignidade. Na amostra em estudo, na escala da mãe, obteve-se um alpha de 0,89 (cuidado); 0,87 (controle). Na escala do pai, obteve-se um alpha de 0,91 (cuidado) e 0,85 (controle).

BSI (Inventário Breve de Sintomatologia - Brief Symptom Inventory) - É uma versão abreviada do Symptom Checklist-90 (SCL-90R), de Derogatis (1993) e consiste em 53 itens, auto respondidos por meio de uma escala Likert, em relação ao grau em que cada sintoma afetou o indivíduo durante a última semana. Os sintomas psicopatológicos são avaliados em nove dimensões: somatização, obsessão-compulsão, sensibilidade interpessoal, depressão ansiedade, hostilidade, ansiedade fóbica, ideação paranoide e psicoticismo. Para avaliar o sofrimento ou aflição psicológica global, foi utilizado o Índice Global de Severidade (IGS). Estudos com a versão original (Derogatis, 1993) indicam boas características 
psicométricas do inventário. A escala possui uma versão em Português, validada por Canavarro (1999) que possui boa confiabilidade. Para uso no Brasil, a versão de Canavarro (1999) foi adaptada pelo grupo de pesquisa e nesta amostra, apresentou valores alpha de 0,96 para a escala total e entre 0,69 e 0,86 para as subescalas.

Procedimentos Éticos, de Coleta e Análise de Dados

O protocolo da pesquisa que originou o banco de dados do qual este estudo é derivado foi aprovado pelo Comitê de Ética em Pesquisa da universidade de origem do projeto. Todos os participantes assinaram - Termo de Consentimento Livre e Esclarecido (TCLE). Os instrumentos foram entregues aos pacientes na $4^{a}$ sessão de psicoterapia em envelope fechado com instruções para seu preenchimento em casa e foram devolvidos na sessão seguinte.

Posteriormente, as informações foram transferidas e organizadas em um banco de dados e as análises do estudo foram realizadas no programa SPSS (Statistical Package for the Social Sciences 22.0). Para estudo da normalidade utilizou-se o teste de KolmogorovSmirnov com correção de Lilliefors. Diante dos resultados, não foram assumidas suposições de normalidade. Foram feitas análises descritivas (média, mínimo e máximo, desvio padrão e percentagens) a fim de caracterizar a amostra. O teste Wilcoxon-Mann Whitney foi utilizado para complementar as análises descritivas, verificando eventuais diferenças nas médias em estudo em pacientes do gênero masculino e feminino. Para medir o relacionamento entre AT, vinculação parental e sintomatologia, utilizou-se o coeficiente de correlação de Spearman. O teste Kruskal Wallis seguido de Post Hoc Dunn foi utilizado para examinar associações entre estilos parentais e AT. O nível de significância adotado foi de $5 \%(p \leq 0,05)$.

\section{Resultados}

Caracterização da Amostra

A amostra foi constituída por 57 pacientes adultos, em PP, sendo 42 mulheres e 15 homens, com idades entre 18 e 62 anos $(M=33,1$ anos; $\mathrm{DP}=13,2$ anos). A maioria possuía ensino superior completo ou incompleto $(71,9 \%)$. Os terapeutas foram 32 mulheres e 2 homens com idades entre 25 e 63 anos ( $M=32,8$ anos; $D P=10,5$ anos). Portanto, mais de um participante era atendido pelo mesmo terapeuta. 
Tabela 1

Distribuição de frequência relativa e absoluta dos participantes (pacientes e terapeutas) de acordo com as características sociodemográficas.

\section{Características Demográficas}

\begin{tabular}{|c|c|c|c|c|c|}
\hline Pacientes $(n=57)$ & $\mathbf{N}$ & $\%$ & Terapeutas $(n=34)$ & $\mathbf{N}$ & $\%$ \\
\hline \multicolumn{3}{|l|}{ Sexo } & \multicolumn{3}{|l|}{ Sexo } \\
\hline Feminino & 46 & & Feminino & 32 & 94,1 \\
\hline Masculino & 15 & & Masculino & 2 & 5,9 \\
\hline \multicolumn{3}{|l|}{ Idade } & \multicolumn{3}{|l|}{ Idade } \\
\hline A té 30 anos & 31 & 54,4 & Até 30 anos & 18 & 52,8 \\
\hline Entre 31 a 50 anos & 16 & 28,1 & Entre 31 a 50 anos & 6 & 17,5 \\
\hline Acima de 50 anos & 10 & 17,5 & Acima de 50 anos & 4 & 11,6 \\
\hline \multicolumn{3}{|l|}{ Escolaridade } & Não respondeu & 6 & 17,6 \\
\hline Ensino médio incompleto & 1 & 1,8 & \multicolumn{3}{|l|}{ Formação } \\
\hline Ensinos médio completo & 8 & 14,0 & Primeiro ano & 12 & 35,3 \\
\hline $\begin{array}{l}\text { Ensino } \\
\text { técnico/profissionalizante }\end{array}$ & 7 & 12,3 & Segundo ano & 4 & 11,8 \\
\hline Ensino superior incompleto & 24 & 42,1 & Terceiro ano & 6 & 17,6 \\
\hline Ensino superior completo & 17 & 29,8 & Corpo clínico & 12 & 35,3 \\
\hline \multicolumn{6}{|l|}{ Estado civil } \\
\hline Solteiro & 36 & 63,2 & & & \\
\hline Casado & 12 & 21,1 & & & \\
\hline Separado/divorciado & 6 & 10,5 & & & \\
\hline União estável & 1 & 1,8 & & & \\
\hline Não respondeu & 2 & 3,5 & & & \\
\hline
\end{tabular}

\section{Análises Descritivas para as Variáveis em Estudo}

Foram realizadas análises descritivas dos resultados dos instrumentos de avaliação de aliança terapêutica (WAI), de vinculação parental (PBI) e de sintomatologia (BSI) na amostra e examinadas possíveis associações com o gênero dos participantes. Com relação aos escores encontrados, verificou-se que, de modo geral, os participantes constituíram boas alianças em terapia, obtendo índices acima do ponto médio da escala (entre 5,6 e 5,9). 
Ao considerar o IGS e as diversas subescalas do BSI, foi possível constatar que os sintomas mais prevalentes na amostra foram os de esfera obsessivo-compulsiva $(M=1,46 \mathrm{DP}=0,89)$, depressiva $(M=1,30$ e $D P=1,00)$ e que indicam sensibilidade interpessoal $(M=1,27$ e $D P=0,95)$. O Índice Global de Severidade apresentou média de 1,11 ( $D P=0,72$ ) numa escala de 0 a 4.

No que concerne aos vínculos parentais, utilizando os pontos de corte para as dimensões do PBI, foi constatado que a maioria dos pacientes referiu baixo cuidado $(59,6 \%)$ e alto controle $(57,9 \%)$ da mãe, bem como baixo cuidado $(63,2 \%)$ e alto controle do pai $(57,9 \%)$. Os resultados descritivos da AT, sintomatologia e vinculação parental são expostos na Tabela 2. 
Tabela 2

Estatísticas descritivas para as escalas totais e subescalas dos instrumentos de aliança terapêutica (WAI), sintomatologia (BSI) e vinculação parental (PBI).

\begin{tabular}{|c|c|c|c|c|}
\hline \multicolumn{5}{|c|}{ Estatisticas descritivas } \\
\hline \multicolumn{5}{|c|}{ WAI } \\
\hline Variáveis em estudo & Minimo & Máximo & Média & Desvio padrăo \\
\hline Total & 2,42 & 6,78 & 5,77 & 0,72 \\
\hline Objetivos & 2,17 & 7,00 & 5,90 & 0,78 \\
\hline Tarefa & 2,92 & 6,83 & 5,81 & 0,68 \\
\hline Vinculo & 2,17 & 6,83 & 5,60 & 0,83 \\
\hline \multicolumn{5}{|c|}{ BSI } \\
\hline Variáveis em estudo & Minimo & Maximo & Média & Desvio padrão \\
\hline Ansiedade & 0,00 & 3,17 & 1,17 & 0,87 \\
\hline Somatizaçăo & 0,00 & 3,57 & 0,83 & 0,87 \\
\hline Psicoticismo & 0,00 & 3,20 & 1,00 & 0,87 \\
\hline Ideaçăo paranoide & 0,00 & 3,80 & 1,20 & 0,95 \\
\hline Obsessão compulsăo & 0,00 & 3,33 & 1,46 & 0,89 \\
\hline Hostilidade & 0,00 & 3,40 & 0,93 & 0,64 \\
\hline Ansiedade fóbica & 0,00 & 3,25 & 0,59 & 0,74 \\
\hline Depressão & 0,00 & 3,67 & 1,30 & 1,00 \\
\hline Sensibilidade interpessoal & 0,00 & 4,00 & 1,27 & 0,95 \\
\hline IGS & 0,08 & 3,27 & 1,10 & 0,72 \\
\hline \multicolumn{5}{|c|}{ PBI } \\
\hline Variáveis em estudo & Minimo & Máximo & Média & Desvio padrāo \\
\hline Mãe cuidado & 3,00 & 36,00 & 22,75 & 8,33 \\
\hline Măe controle & 0,00 & 34,00 & 16,31 & 8,42 \\
\hline Pai cuidado & 2,00 & 36,00 & 19,61 & 9,46 \\
\hline Pai controle & 0,00 & 36,00 & 14,80 & 8,19 \\
\hline
\end{tabular}

Nota: WAI $=$ Working Alliance Inventory; PBI $=$ Parental bonding Instrument; BSI = Brief Symptom Inventory.

A partir das combinações das duas dimensões do PBI, os estilos parentais: "cuidado ótimo", "negligente", "controle afetivo" e 
"controle sem afeto" foram identificados. Foi constatado que o estilo mais prevalente na amostra foi o de "controle sem afeto" tanto para a mãe como para o pai. Os resultados estão expostos na Tabela 3.

\section{Tabela 3}

Frequência relativa e absoluta para os estilos parentais na amostra em estudo $(n=57)$

\begin{tabular}{lcccc}
\hline \multirow{2}{*}{ Estilos parentais } & \multicolumn{2}{c}{ Estilo parental Mãe } & \multicolumn{2}{c}{ Estilo parental Pai } \\
\cline { 2 - 5 } & $\mathrm{N}$ & $\%$ & $\mathrm{~N}$ & $\%$ \\
\cline { 2 - 5 } Cuidado ótimo & 11 & 19,3 & 11 & 19,3 \\
Negligente & 13 & 22,8 & 14 & 24,6 \\
Controle afetivo & 13 & 22,8 & 11 & 19,3 \\
Controle sem afeto & 20 & 35,1 & 21 & 36,8 \\
Total & 57 & 100,0 & 57 & 100,0 \\
\hline
\end{tabular}

No que diz respeito às possíveis diferenças com relação ao gênero dos participantes, através do teste Wilcoxon-Mann Whitney, verificouse que a dimensão controle ou superproteção do pai mostrou diferença entre as médias dos rankings das mulheres $(n=42$; $M=31,5)$ e dos homens $(n=15 ; M=21,7)$ sendo significativamente maior o controle na amostra feminina $(U=206,5 ; W=306,5 p=$ $0,049)$.

Análise de Correlação para Aliança Terapêutica, Vinculação Parental e Sintomas dos Pacientes

Foram avaliadas possíveis associações entre AT (WAI total e subescalas), vinculação parental (subescalas da mãe e do pai e estilos parentais de ambos) e sintomatologia (IGS e todas as nove subescalas do BSI) através do teste de correlação ordinal de Spearman. Como estas análises envolveram diversas subescalas, somente os valores das associações significativas são descritos.

AT e vinculação Parental: Foram encontradas correlações positivas significativas entre o cuidado da mãe e o escore médio total do WAI $\left(r_{s}=0,352 ; p=0,007\right)$, bem como com as subescalas tarefas $\left(r_{s}=\right.$ $0,295 ; p=0,026)$, vínculo $\left(r_{s}=0,268 p=0,044\right)$ e objetivos $\left(r_{s}=\right.$ $0,358 \mathrm{p}=0,006)$ deste instrumento. O controle da mãe mostrou associação negativa com os objetivos da terapia $\left(r_{s}=-0,275 ; p=\right.$ $0,039)$. 
Possíveis associações entre estilos parentais e AT foram investigadas por meio de teste Kruskall Wallis. Verificou-se que houve associação entre o estilo parental da mãe e a subescala de objetivos do WAI $\left[\mathrm{X}^{2} \mathrm{KW}(3)=9,754, \mathrm{p}=0,021, \mathrm{n}=57\right]$ bem como o total do WAI $\left[\mathrm{X}^{2} \mathrm{kw}(3)=8,846, \mathrm{p}=0,031, \mathrm{n}=57\right]$. O Teste Post Hoc de Dunn mostrou que as diferenças encontradas na amostra ocorreram entre os estilos "cuidado ótimo" e "controle sem afeto" da mãe. A AT total foi significativamente maior nos participantes com "cuidado ótimo" da mãe em comparação aos que relataram "controle sem afeto" visto que, a diferença entre os rankings médios de 18,166 foi estatisticamente significativa ao nível de 0,021. Pacientes com mães com "cuidado ótimo" também apresentaram índices mais elevados nos objetivos da terapia, em comparação aos que receberam "controle sem afeto". A diferença entre os rankings médios de 18,984 foi estatisticamente significativa ao nível de 0,014.

AT e sintomatologia: Não foram encontradas associações significativas.

Vinculação parental e sintomatologia: $O$ cuidado da mãe foi negativamente correlacionado com depressão $\left(r_{\mathrm{s}}=-0,342 ; \mathrm{p}=\right.$ $0,009)$, e com o IGS, medida de severidade global de sintomas $\left(r_{s}=-\right.$ $0,273 \mathrm{p}=0,040)$. Já na escala do pai, a dimensão controle apresentou relação positiva com o IGS $\left(r_{s}=0,362 ; p=0,006\right)$ e com sintomas das dimensões somatização $\left(r_{s}=0,276 ; p=0,038\right)$; psicoticismo $\left(r_{s}=0,289 ; p=0,029\right)$; Ideação paranoide $\left(r_{s}=0,321\right.$; $\mathrm{p}=0,015)$; hostilidade $\left(r_{s}=0,368 \mathrm{p}=0,005\right)$; depressão $\left(r_{s}=0,274\right.$ $p=0,039)$; sensibilidade interpessoal $\left(r_{s}=0,347 \mathrm{p}=0,008\right)$; e ansiedade $\left(r_{s}=0,356 p=0,007\right)$.

\section{Discussão}

Este estudo procurou investigar relações entre aliança terapêutica, memórias de vinculação parental e sintomatologia atual de pacientes adultos que estavam na fase inicial de PP. De modo geral, os resultados indicam que existem associações entre as variáveis, evidenciando o caráter complexo dos processos que ocorrem em psicoterapia. O grupo de pacientes avaliado estava iniciando PP e caracterizou-se por uma maioria do sexo feminino, com alta escolaridade e sintomas psicológicos diversos, com destaque para aqueles que compõem as dimensões: obsessivo compulsiva, depressão e sensibilidade interpessoal.

Os Escores de Aliança Terapêutica e a Vinculação Parental

Os índices de AT encontrados na amostra indicam bons níveis de vinculação com o terapeuta, compatíveis com o esperado na fase 
inicial de uma psicoterapia (Luz, 2005). Partindo do pressuposto de que vínculos com os pais em etapas precoces do desenvolvimento podem afetar o relacionamento atual com o terapeuta, foi verificada associação entre a AT e vínculo parental. Os achados indicam que a AT é maior nos pacientes que relataram ter recebido mais cuidados de suas mães. Possivelmente, internalizar a figura da mãe como alguém que despende cuidados e é afetuosa, favoreça a confiança nos relacionamentos e, por conseguinte, favoreça a confiança e a colaboração com o terapeuta.

Entretanto, não foi encontrada correlação entre AT e cuidado do pai. Considerando que a amostra do estudo é predominantemente feminina e que a psicoterapia é praticada mais por mulheres do que homens é possível pressupor que o gênero do terapeuta seja uma variável interveniente, visto que $94 \%$ dos profissionais desta amostra são mulheres.

Neste estudo, os estilos parentais da mãe estavam relacionados em particular com os objetivos do tratamento e com o escore total de aliança. Pacientes com mães que exerceram um cuidado ótimo tinham uma melhor qualidade destas dimensões da AT em comparação com aqueles cujas mães exerceram controle sem afeto. Este dado sugere que a aliança inicial com o terapeuta tem relação com a história de vinculação passada do paciente, já que a relação terapêutica possui elementos relacionados com esta base segura e com as figuras de apego primárias (Bowlby, 1984/2002; Mikulincer \& Shaver, 2012). Além disso, ressalta-se que o estilo parental controle sem afeto da mãe pode estar associado à dificuldade em construir as metas no início da terapia, visto que, na população geral, a superproteção tem relação com falta de autonomia na vida adulta (Otani, Suzuki, Matsumoto, \& Kamata, 2009).

Uma variável que pode estar mediando o efeito dos padrões estabelecidos no passado, no processo de vinculação na terapia e que não foi foco deste estudo, pode ser justamente, a postura do terapeuta. Pesquisas indicam que terapeutas considerados mais empáticos e mais envolvidos, e menos passivos e indiferentes impactam positivamente a aliança (Hersoug, Hoglend, Havik, Lippe \& Monsen, 2009). Isso pode ter sido característico desta amostra.

Atentar para o relacionamento terapêutico e a possível repercussão dos vínculos parentais no momento inicial da psicoterapia pode ser útil para identificar e resolver rupturas nesta relação (Safran, Muran \& Eubanks-Carter, 2011). Estudos suportam esta discussão ao demonstrar que a resolução de rupturas no relacionamento está relacionada com melhores resultados em psicoterapia (Safran et al., 2011) e com diminuição de sintomas (Strauss et al., 2006). Ademais, Hersoug, Hoglend, Monsen e Havik (2001) ressaltam que depois de ser estabelecida a AT, o impacto das experiências passadas diminui, podendo indicar que o relacionamento terapêutico pode ser um 
regulador importante de características do paciente que poderiam afetar a AT. Este dado revela que a relação não é "unilateral" mas que é antes uma complexa interação.

\section{Aliança Terapêutica e Sintomatologia dos Pacientes}

Os achados do presente estudo corroboram o estudo de Gomes (2003), de que não foram encontradas relações entre a AT e os sintomas dos pacientes em PP. Embora a AT seja um preditor significativo de resultados em terapia, a sua especifica relação com a intensidade ou frequência de sintomas ainda é bastante discutida. Estudos recentes reforçam a compreensão de um modelo de relação causal recíproca, ao invés de unidirecional. Ou seja, a AT parece ser preditora de diminuição de sintomas ao mesmo tempo em que a mudança nos sintomas parece afetar a AT, o que consequentemente tem relação com os resultados da terapia (Falkenström, Granström \& Holmqvist, 2013; Xu \& Tracey, 2015). Nesse sentido, considera-se que um delineamento transversal não foi sensível à complexa relação destas variáveis, sendo necessária uma investigação longitudinal para compreender as interações e variações ao longo do tratamento.

Experiências de Vinculação e sua Associação com os Sintomas na Vida Adulta

Conforme esperado, encontrou-se associação entre o modo como ambos os pais interagem com seus filhos na infância e adolescência e a intensidade de sintomas diversos na vida adulta. A depressão estava associada com índices baixos de cuidado da mãe e com a superproteção do pai. Campos et al. (2010) ressaltam que relacionamentos precoces, caracterizados por altos níveis de superproteção estão associados com depressão em adultos. Ademais, foram encontradas relações entre o controle ou superproteção do pai e a sensibilidade interpessoal dos pacientes. Estudos anteriores sustentam parcialmente este resultado, visto que memórias acerca de pais controladores também estavam associadas com esta sintomatologia em pacientes depressivos (Avagianou \& Zafiropolou, 2008; Johnstone et al., 2013).

É importante destacar que o presente estudo foi realizado com uma amostra naturalística $e$, portanto, heterogênea em termos de sintomatologia. Neste grupo, a dimensão controle do pai foi a que apresentou relação com o maior número de sintomas e, inclusive, como índice global do BSI, considerado uma medida de sofrimento geral. Nota-se que o controle do pai foi significativamente maior na amostra feminina, mas que as memórias acerca de pais superprotetores ou controladores estavam relacionados com maiores índices sintomatológicos de ambos os gêneros. 
De modo geral, este estudo demonstrou que a história pregressa de vinculação dos pacientes em psicoterapia está associada tanto ao seu quadro sintomático (que por vezes os levam ao tratamento) como ao modo como o paciente se relaciona com o terapeuta, estabelecendo uma aliança de trabalho, em especial a capacidade de estabelecer acordos sobre metas a serem atingidas (objetivos). Esse é um achado relevante na medida em que pesquisas têm apontado que 0 desacordo entre paciente e terapeuta sobre o que deve ser atingido como resultado do tratamento é um dos fatores que podem explicar o abandono precoce em PP (Jung, Serralta, Nunes \& Eizirik, 2013; Jung, Serralta, Nunes \& Eizirik, 2015).

Tomados em conjunto, os achados do presente estudo enfatizam o importante papel que a relação entre pais e filhos executa no desenvolvimento posterior dos indivíduos (Bowlby, 1984/2002; Mikulincer \& Shaver, 2012) e seus efeitos na AT (Hersoug et al., 2002; Tereno et al., 2008). Logo, as experiências passadas de vinculação são de grande importância para compreender as variações na aliança e na expressão de sintomas diversos.

\section{Conclusão}

Este artigo ofereceu diversas contribuições para a compreensão das relações entre AT, experiências de vinculação com os pais na infância e adolescência e sintomatologia de pacientes adultos em PP. Ainda que se trate de um estudo com abordagem correlacional, os achados estão de acordo com os pressupostos teóricos da teoria do apego e com dados empíricos de estudos internacionais que examinaram estas relações no contexto da psicoterapia.

Sendo assim, os resultados encontrados podem contribuir para adaptar intervenções às demandas do paciente em psicoterapia e instruir terapeutas a atentar para a história relacional de seus pacientes e a intensidade de sintomas, variáveis que podem afetar, direta ou indiretamente, a qualidade da AT inicial e, consequentemente, o progresso do paciente no tratamento. Ademais, considerando a escassez de estudos nacionais sobre AT e que a pesquisa empírica psicanalítica é ainda incipiente no país, pretende-se com o estudo encorajar os terapeutas a utilizar medidas mais objetivas para avaliar os processos e características da psicoterapia, como uma ferramenta possível e relevante na pratica clínica.

Sugere-se que estudos subsequentes sejam realizados com uma amostra maior de pacientes para examinar, por meio de delineamentos explicativos (com análises de regressão múltipla e/ou modelagem de equações estruturais) a interação entre AT, vinculação parental e sintomatologia. Estudos longitudinais também podem auxiliar na compreensão destas relações. 


\section{Referências}

Abreu, C. N. (2005a). Teoria do apego: fundamentos, pesquisas e implicações clínicas. São Paulo: Casa do Psicólogo.

Abreu, C. N. (2005b). A teoria da vinculação e a prática da psicoterapia cognitiva. Revista Brasileira de Terapias Cognitivas, 1(2), 43-58. Retrieved from http://pepsic.bvsalud.org/scielo.php?script=sci_arttext\&pid=S1 80856872005000200005

Ainsworth, M. D. S., Blehar, M. C., Waters, E., \& Wall, S. (1978/2001). Patterns of attachment: A psychological study of the strange situation. New York: Psychology Press.

Arnow, B. A., Steidtmann, D., Blasey, C., Manber, R., Constantino, M. J., Klein, D. N., \& Kocsis, J. H. (2013). The relationship between the therapeutic alliance and treatment outcome in two distinct psychotherapies for chronic depression. Journal of Consulting and Clinical Psychology, 81(4), 627-638. doi: 10.1037/a0031530.

Avagianou, P. A., \& Zafiropoulou, M. (2008). Parental bonding and depression: Personality as a mediating factor. International J ournal of Adolescent Medicine and Health, 20(3), 261-270. doi: 10.1515/ijamh.2008.20.3.261.

Bordin, E. S. (1979). The Generalizability of Psychoanalitic Concept of the Working Alliance. Psychoterapy: Theory, Research, and Practice, 16(3), 252-260. doi: 10.1037/h0085885.

Bordin, E. S. (1994). Theory and research on the therapeutic working alliance: new directions. In A. O. Horvath \& L. S. Greenberg (Eds.), Wiley series on personality processes. The working alliance: Theory, research, and practice (pp. 13-37). Manhattan: John Wiley \& Sons.

Bowlby, J. (1984/2002). Apego: a natureza do vínculo (Vol. 1). São Paulo: Martins Fontes.

Campos, R. C., Besser, A., \& Blatt, S. J. (2010). The mediating role of self-criticism and dependency in the association between perceptions of maternal caring and depressive symptoms. Depression and Anxiety, 27(12), 1149-1157. doi: 10.1002/da.20763.

Canavarro, M. C. S. (1999). Inventário de Sintomas Psicopatológicos: BSI. In M. R. Simões, M. S. Gonçalves, \& L. S. Almeida (Eds.), Testes e provas psicológicas em Portugal (Vol. 2, pp. 87-109). Braga: Sistemas Humano e Organizacionais.

Degnan, A., Seymour-Hyde, A., Harris, A., \& Barry, K. (2014). The Role of Therapist Attachment in Alliance and Outcome: A Systematic Literature Review. Clinical Psychology \& Psychotherapy, 23(1), 47-65. doi: 10.1002/cpp.1937. 
Derogatis, L. R. (1993). BSI: Administration, scoring and procedures manual for the Brief Symptom Inventory (3nd ed.). Minneapolis: National Computer Systems.

Diener, M. J., \& Monroe, J. M. (2011). The relationship between adult attachment style and therapeutic alliance in individual psychotherapy: a meta-analytic review. Psychotherapy, 48(3), 237-248. doi: 10.1037/a0022425.

Eizirik, C. L., Libermann, Z., \& Costa, F. (2008). A relação terapêutica: transferência, contratransferência e aliança terapêutica. In A. V. Cordioli, (Ed.), Psicoterapias: abordagens atuais (pp. 74-84). Porto Alegre: Artmed.

Falkenström, F., Granström, F., \& Holmqvist, R. (2013). Therapeutic alliance predicts symptomatic improvement session by session. Journal of Counseling Psychology, 60(3), 317-328. doi: $10.1037 / \mathrm{a} 0032258$.

Fortuna, K., \& Roisman, G. I. (2008). Insecurity, stress, and symptoms of psychopathology: Contrasting results from selfreports versus interviews of adult attachment. Attachment \& Human Development, 10(1), 11-28. doi: $10.1080 / 14616730701868571$.

Freud, S. (1912/1976). A dinâmica da transferência. In J. Salomão (Ed.), Edição standard brasileira das obras psicológicas completas de Sigmund Freud (Vol. 12, pp. 131-143). Rio de Janeiro: Imago.

Gao, Y., Raine, A., Chan, F., Venables, P. H., \& Mednick, S. A. (2010). Early maternal and paternal bonding, childhood physical abuse and adult psychopathic personality. Psychological Medicine, 40(6), 1007-1016. doi: $10.1017 /$ S0033291709991279.

Greenson, R. R. (1965/2008). The working alliance and the transference neurosis. The Psychoanalytic Quarterly, 77(1), 77102. doi: $10.1002 / j .2167-4086.2008 . t b 00334$.

Gomes, F. G. (2003). A relação entre os mecanismos de defesa e a qualidade da aliança terapêutica em psicoterapia de orientação analítica de adultos: um estudo exploratório. (Dissertação de mestrado não publicada). Universidade Federal do Rio Grande do Sul, RS, Brasil. Retrived from http://ethos.bl.uk/OrderDetails.do?uin=uk.bl.ethos. 576108

Hauck, S., Schestatsky, S., Terra, L., Knijnik, L., Sanchez, P., \& Ceitlin, L. H. F. (2006). Adaptação transcultural para o português brasileiro do Parental Bonding Instrument (PBI). Revista de Psiquiatria do Rio Grande do Sul, 28(2), 162-168. Retrieved from http://www.scielo.br/scielo.php?script=sci_arttext\&pid=S0101$81082006000200008 \& \operatorname{lng}=$ en\&tIng=pt. 
Hersoug, A. G., Hoglend, P., Monsen, J. T., \& Havik, O. E. (2001). Quality of working alliance in psychotherapy. Journal of Psychotherapy, Practice and Research, 10(4), 205-2016. Retrieved from http://www.ncbi.nlm.nih.gov/pmc/articles/PMC3330657/pdf/20 5.pdf

Hersoug, A. G., Hoglend, P., Havik, O. E., Lippe, A, \& Monsen, J. (2009). Therapist characteristics influencing the quality of alliance in long-term psychotherapy. Clinical Psychology \& Psychotherapy, 16(2), 100-110. doi: 10.1002/cpp.605.

Hofmann, S. G., Asnaani, A., Vonk, I. J. J., Sawyer, A. T., \& Fang, A. (2012). The efficacy of cognitive behavioral therapy: a review of meta-analyses. Cognitive Therapy and Research, 36(5), 427440. doi: $10.1007 / \mathrm{s} 10608-012-9476-1$.

Holmes, J. (2014). The search for the secure base: Attachment theory and psychotherapy. London: Routledge.

Horvath, A. O., \& Greenberg, L. S. (1994). The Working Alliance: Theory, Research and Practice. New York: John Wiley \& Sons.

Horvath, A. O., Del Re, A. C., Flückiger, C., \& Symonds, D. (2011). Alliance in individual psychotherapy. Psychotherapy, 48(1), 916. doi: $10.1037 / a 0022186$.

Johnstone, J. M., Carter, J. D., Luty, S. E., Mulder, R. T., Frampton, C. M., \& Joyce, P. R. (2013). Maternal care and paternal protection influence response to psychotherapy treatment for adult depression. Journal of Affective Disorders, 149(1), 221229. doi: 10.1016/j.jad.2013.01.030.

Jung, S. I., Serralta F. B., Nunes M. L., \& Eizirik C. L. (2013). Beginning and end of treatment of patients who dropped out of psychoanalytic psychotherapy. Trends in Psychiatry and Psychotherapy, 35(3), 181-190. Retrieved from http://dx.doi.org/10.1590/S2237-60892013000300005.

Jung, S. I., Serralta F. B., Nunes M. L. T., \& Eizirik C. L. (2015). Desistência e Conclusão em Psicoterapia Psicanalítica, um estudo qualitativo de pacientes de Porto Alegre, Brasil. Revista Brasileira de Psicoterapia, 17(1), 25-40. Retrieved from http://rbp.celg.org.br/detalhe_artigo.asp?id=167.

Leichsenring, F., \& Rabung, S. (2011). Long-term psychodynamic psychotherapy in complex mental disorders: update of a metaanalysis. The British Journal of Psychiatry, 199(1), 15-22. doi: 10.1192/bjp.bp.110.082776.

Lewis, J. M. (2000). Repairing the bond in important relationships: A dynamic for personality maturation. American Journal of Psychiatry, 157(9), 1375-1378. Retrieved from http://dx.doi.org/10.1176/appi.ajp.157.9.1375.

Lima, A. R., Mello, M. F., \& Mari, J. J. (2010). The role of early parental bonding in the development of psychiatric symptoms in 
adulthood. Current Opinion in Psychiatry, 23(4), 383-387. doi: 10.1097/YCO.0b013e32833a51ce.

Luz, A. B. (2005). Fases da psicoterapia. In C. L. Eizirik, R. Aguiar, \& S. Schestatski (Eds.), Psicoterapia de Orientação Analítica: Fundamentos teóricos e clínicos (pp. 254-267). Porto Alegre: Artmed.

Marmarosh, C. L., Gelso, C. J., Markin, R. D., Majors, R., Mallery, C., \& Choi, J. (2009). The real relationship in psychotherapy: Relationships to adult attachments, working alliance, transference, and therapy outcome. Journal of Counseling Psychology, 56(3), 337-350.doi: 10.1037/a0015169.

Mikulincer, M., \& Shaver, P. R. (2012). An attachment perspective on psychopathology. World Psychiatry, 11(1), 11-15. doi: 10.1016/j.wpsyc.2012.01.003.

Morgan, C., \& Fisher, H. (2007). Environment and schizophrenia: childhood trauma, a critical review. Schizophrenia Bulletin, 33(1), 3-10. doi: 10.1093/schbul/sbl053.

Otani, K., Suzuki, A., Matsumoto, Y., \& Kamata, M. (2009). Parental overprotection increases interpersonal sensitivity in healthy subjects. Comprehensive Psychiatry, 50(1), 54-57. doi: 10.1016/j.comppsych.2008.05.009

Parker, G., Tupling, H., \& Brown, L. B. (1979/2011). A parental bonding instrument. British Journal of Medical Psychology, 52(1), 1-10. doi: 10.1111/j.2044-8341.1979.tb02487.

Safran, J. D., Muran, J. C., \& Eubanks-Carter, C. (2011). Repairing alliance ruptures. Psychotherapy, 48(1), 80-87. doi: $10.1037 / \mathrm{a} 0022140$.

Spokes, N. (2012). Client non-disclosure in treatment for eating disorders and its relationship with current symptoms: the roles of shame, the therapeutic alliance, and therapist self-disclosure. (Tese de doutorado não publicada). Retrieved from http://ethos.bl.uk/OrderDetails.do?uin=uk.bl.ethos.576108

Strauss, J. L., Hayes, A. M., Johnson, S. L., Newman, C. F., Brown, J. K., Barber, J. P., ... Beck, A. T. (2006). Early alliance, alliance ruptures, and symptom change in a nonrandomized trial of cognitive therapy for avoidant and obsessive-compulsive personality disorders. Journal of Consulting and Clinical Psychology, 74(2), 337-345. doi: 10.1037/0022006X.74.2.337.

Tereno, S., Soares, I., Martins, C., Celani, M., \& Sampaio, D. (2008). Attachment styles, memories of parental rearing and therapeutic bond: A study with eating disordered patients, their parents and therapists. European Eating Disorders Review, 16(1), 49-58.doi: 10.1002/erv.801. 
Zetzel, E. R. (1956). Current concepts of transference. International Journal of Psychoanalysis, 37(4-5), 369-376. Retrieved from http://www.ncbi.nlm.nih.gov/pubmed/13366506.

$\mathrm{Xu}, \mathrm{H} .$, \& Tracey, T J. (2015). Reciprocal influence model of working alliance and therapeutic outcome over individual therapy course. Journal of Counseling Psychology, 62(3), 351-359. Retrieved from http://dx.doi.org/10.1037/cou0000089.

\section{Endereço para correspondência}

\section{Carolina Palmeiro Lima}

Universidade do Vale do Rio dos Sinos - UNISINOS

Avenida Unisinos, 950, Cristo Rei, CEP 93022-750, São Leopoldo - RS, Brasil

Endereço eletrônico: psi.carolinalima@gmail.com

Fernanda Barcellos Serralta

Universidade do Vale do Rio dos Sinos - UNISINOS

Avenida Unisinos, 950, Cristo Rei, CEP 93022-750, São Leopoldo - RS, Brasil

Endereço eletrônico: fernandaserralta@gmail.com

Recebido em: 04/01/2016

Aceito em: 04/04/2017

\section{Notas}

* Psicóloga (UNISINOS). Mestranda em Psicologia, Universidade Federal do Rio Grande do Sul (UFRGS).

** Psicóloga, Doutora em Psiquiatria. Docente do Programa de Pós Graduação em Psicologia, Universidade do Vale do rio dos Sinos (UNISINOS).

Este artigo de revista Estudos e Pesquisas em Psicologia é licenciado sob uma Licença Creative Commons Atribuição-Não Comercial 3.0 Não Adaptada. 